\title{
Finding a niche: studies from the Drosophila ovary
}

Susan Eliazer and Michael Buszczak*

\begin{abstract}
Specialized microenvironments called niches help maintain stem cells in an undifferentiated and selfrenewing state. The existence of niches has long been predicted from mammalian studies, but identifying stem cells in their native environments in vivo has remained a challenge in most vertebrates. Many of the mechanistic insights into how niches regulate stem cell maintenance have been obtained using invertebrate models such as Drosophila. Here, we focus on the Drosophila ovarian germline stem cell niche and review recent studies that have begun to reveal how intricate crosstalk between various signaling pathways regulates stem cell maintenance, how the extracellular matrix modulates the signaling output of the niche and how epigenetic programming influences cell development and function both inside and outside the niche to ensure proper tissue homeostasis. These insights will probably inform the study of mammalian niches and how their malfunction contributes to human disease.
\end{abstract}

\section{Introduction}

Stem cells are essential for tissue homeostasis, particularly in organs that exhibit high rates of cellular turnover such as the skin, intestine and hematopoietic system. Without the self-renewing capacity of stem cells, these tissues quickly cease to function properly, leading to various conditions including infertility, anemia and immunodeficiency. Overproliferation of stem cells is equally undesirable and can disrupt normal tissue homeostasis, possibly contributing to tumor formation and growth. Interestingly, cells within tumors often exhibit a hierarchy of malignant potential, giving rise to the notion that small populations of cancer stem cells may be responsible for propagating certain cancers $[1,2]$. Prospectively identifying these cells and determining how they differ from their normal stem cell counterparts

*Correspondence: michael.buszczak@utsouthwestern.edu

Department of Molecular Biology, University of Texas Southwestern Medical Center at Dallas, Dallas, TX 75390-9148, USA will probably provide important insights into the origin and progression of malignancy.

The concept of the cellular niche represents one of the central paradigms in stem cell biology. First proposed by Schofield in 1978 [3], the niche hypothesis posits that specific locations or microenvironments within tissues prevent the maturation of resident stem cells. The niche model is consistent with many observations made in mammalian cell transplantation experiments, but difficulties in unequivocally identifying individual stem cells within their native environment prevented further testing of this hypothesis. Twenty years following Schofield's seminal publication, Xie and Spradling provided compelling experimental evidence that a cellular niche supports the maintenance of germline stem cells (GSCs) in the Drosophila adult ovary [4]. Shortly thereafter, similar findings were reported in the Drosophila testis [5,6]. Taken together, the study of the Drosophila ovary and testis has greatly enhanced our understanding of the basic principles that govern niche formation and function. Several recent publications have reviewed studies of stem cells within the testis $[7,8]$. Here we will focus on reviewing work describing the formation and regulation of the ovarian stem cell niche.

\section{Organization of the adult Drosophila ovary}

Drosophila females have two ovaries typically comprised of 16 to 21 tube-like structures called ovarioles [9]. Each ovariole contains six to eight sequentially developing egg chambers, each of which is initially assembled in a structure at the tip of the ovariole called the germarium (Figure 1). Two to three GSCs reside at the anterior tip of the germarium immediately adjacent to the niche, which includes a small cluster of five to seven cap cells attached to eight to 10 terminal filament cells. GSCs typically undergo asymmetric self-renewing divisions, producing one daughter stem cell that remains associated with the cap cell niche and a second daughter that is displaced away from the niche and as a result differentiates. This newly formed cystoblast undergoes four incomplete mitotic divisions to form an interconnected 16-cell cyst.

Escort cells, also called inner sheath cells or inner germarium sheath cells, line the anterior region of the germarium and send extensions between germline cysts during the earliest stages of their differentiation. Recent 
(a)

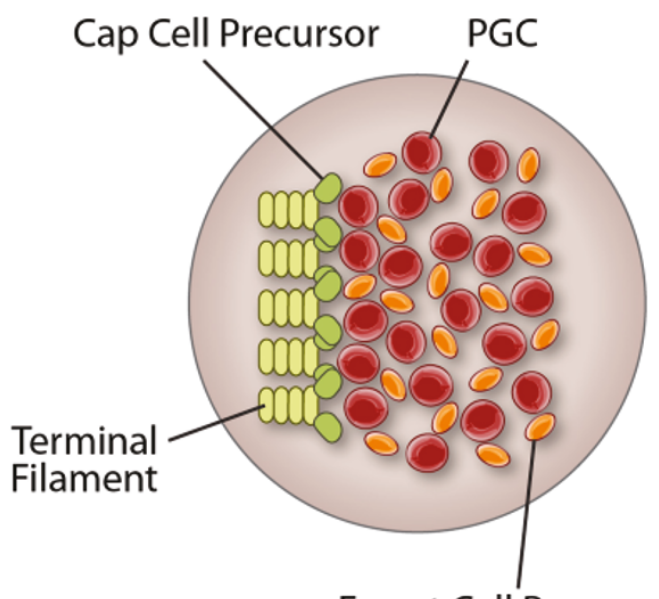

(b)

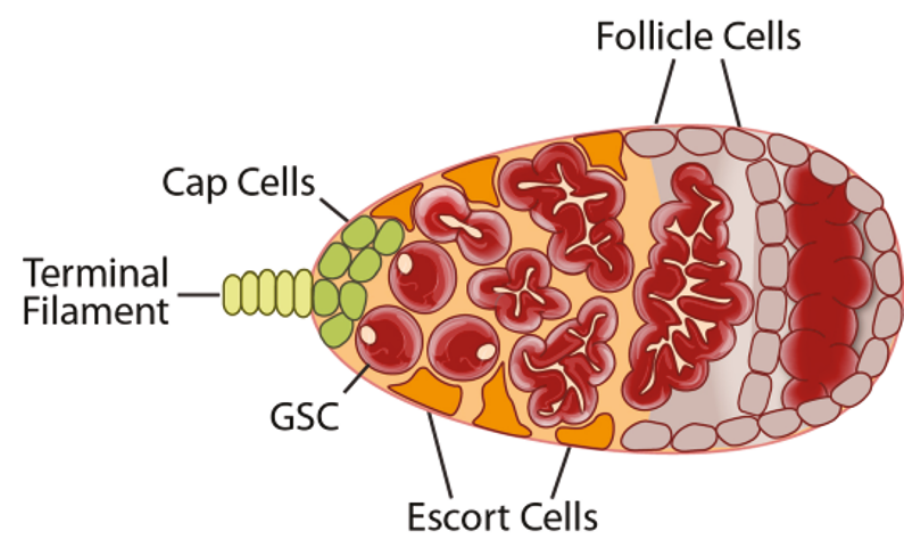

\section{Escort Cell Precursor}

Figure 1. Organization of the developing female gonad and the adult germarium. (a) By the end of larval development, approximately 100 primordial germ cells (PGCs) (red) populate the gonad and associate with cap cell precursor (dark green) and escort cell precursor cells (orange). Terminal filament stacks (light green) begin to form and signal to adjacent somatic cells through the Delta-Notch pathway, inducing them to become cap cells. (b) The differentiation of adult germline cells (red) can be traced based on morphological changes in the fusome (beige), an endoplasmic reticulum-like organelle that appears round in the germline stem cells (GSCs) and becomes increasingly more branched as germline cysts develop [76]. Adult GSCs reside in a niche formed by the terminal filament (light green) and cap cells (dark green). Escort cells (orange) help to guide developing cysts as they pass through the germarium. Eventually a single layer of follicle cells (grey) surrounds the germline cysts and these enveloped cysts bud off the germarium to form an egg chamber.

live imaging experiments show that these escort cells help maturing germline cysts move posteriorly through the germarium [10]. Eventually progeny of two follicle stem cells envelop the 16-cell germline cyst, and together this cluster of cells buds off from the germarium to form an egg chamber.

Many of the aforementioned cell types can be identified at single-cell resolution based on the architecture of the germarium and through the use of morphological and molecular markers. The ability to distinguish individual cells within their native environment, coupled with the ability to genetically manipulate these cells, makes the Drosophila germarium a powerful platform with which to dissect the molecular mechanisms governing stem cell maintenance.

\section{Bone morphogenetic protein signaling in the adult germline stem cell niche}

Significant progress has been made in defining the signaling events that promote GSC self-renewal (Figure 2). One of the principle ligands required for GSC maintenance is Decapentaplegic (Dpp), a member of the bone morphogenetic protein (BMP) superfamily of signaling molecules [11]. Glass bottom boat (Gbb), a BMP5/6/7/8 homolog [12], also functions to support GSC maintenance [13]. Disruption of either $d p p$ or $g b b$ results in GSC loss, while overexpression of $d p p$, but not $g b b$, causes a GSC tumor phenotype. RT-PCR analysis of isolated cells suggests that several different subpopulations of somatic cells at the anterior tip of the germarium express $d p p$ and $g b b$ [13]. In situ hybridization also detects $d p p$ transcripts within this region $[4,14,15]$.

BMP ligand produced at the anterior tip of the germarium transduces its effects through the type I receptors Thickveins and Saxophone and the type II receptor Punt. Genetic mosaic experiments show that these receptors function autonomously in GSCs and are necessary for their maintenance $[11,16]$. Activation of the receptor complex results in phosphorylation of Mothers Against Dpp (Mad), which then binds to its partner Medea [17] and translocates into the nucleus. Phosphorylated Mad and Medea associate with a specific silencer element in the promoter of the bag of marbles (bam) gene and repress its transcription $[13,18,19]$. Bam expression is both necessary and sufficient for germline differentiation [20-22]. Loss of bam results in germline tumors that contain undifferentiated cells that exist in a pre-cystoblast state, whereas misexpression of bam in GSCs results in their precocious differentiation.

BMP pathway activation also results in high levels of Daughters against $d p p$ (Dad) expression in GSCs $[13,23,24]$. In GSC daughters displaced away from the cap cells, Dad expression decreases whereas bam transcription increases. Remarkably, this switch in Dad and bam expression occurs one cell diameter away from the cap cells. Several studies have begun to describe some of 


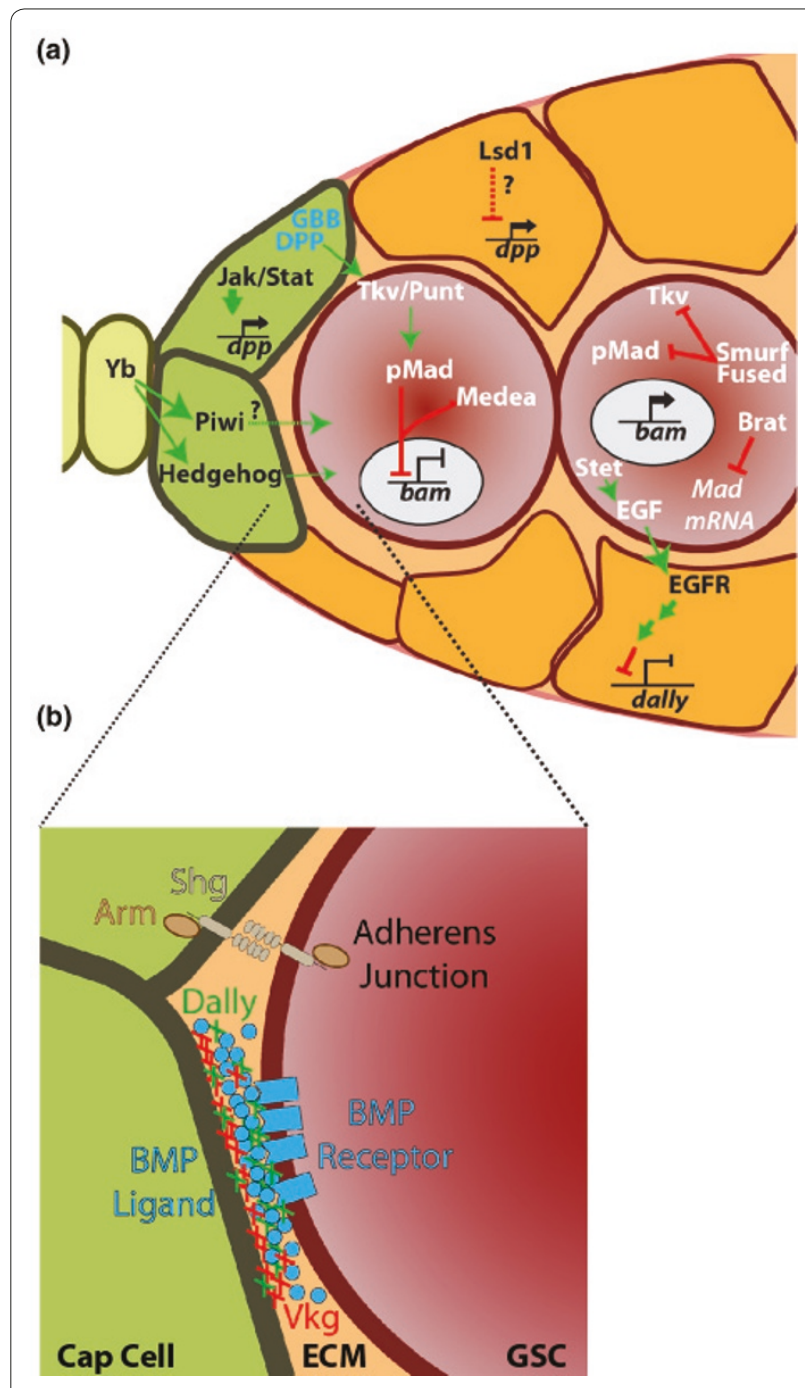

Figure 2. Signaling within the female germline stem cell niche. (a) Schematic illustrating that Decapentaplegic (Dpp) and Glass bottom boat (Gbb) produced in the anterior of the germarium binds to heterodimeric receptors on the surface of germline stem cells (GSCs). Activation of the receptor results in phosphorylation of Mad (pMad) which then partners with Medea and translocates into the nucleus, where it directly represses the transcription of bag of marbles (bam). This repression is relieved once a GSC daughter leaves the cap cell niche. Smurf, Fused, Brain tumor (Brat) and miR184 all act to rapidly reduce bone morphogenetic protein (BMP) responsiveness within the cystoblast. Niche signaling is limited to the anterior of the germarium by $L s d 1$, which represses $d p p$ expression outside the normal niche and by epidermal growth factor (Egf) signaling from the germline, which serves to limit dally expression in the escort cells. EGFR, epidermal growth factor receptor; JAKJSTAT, Janus kinase/signal transducer and activator of transcription; pMad, phosphorylated Mothers Against Dpp; Tkv, Thickveins; YB, Female sterile (1) Yb. (b) Components of the extracellular matrix (ECM), including Viking (Vkg; red) and Division abnormally delayed (Dally; green) help to stabilize and limit BMP ligands (blue circles) within the anterior of the germarium. The adherens junction proteins Armadillo (Arm; brown) and Shotgun (Shg; grey) promote cell-cell adhesion between the cap cells (green) and GSCs (dark red). the intrinsic mechanisms responsible for this sharp gradient of BMP responsiveness. During Drosophila embryogenesis, the E3 ubiquitin ligase Smurf has been shown to oppose BMP signaling by targeting Mad for degradation [25]. Consistent with these observations, Smurf mutants also display greater Dpp responsiveness within the germline [23]. A recent study describes how Smurf partners with the serine/threonine kinase Fused to antagonize BMP signaling within cystoblasts and differentiating cysts by promoting the degradation of Thickveins [26]. In addition, the translational regulator Brain Tumor (Brat) acts as a germline differentiation factor and represses both Mad and $d M y c$ [27]. Lastly, mir-184 appears to regulate Saxophone levels within the cystoblast [16].

These findings suggest that multiple mechanisms ensure a very rapid downregulation of Dpp responsiveness in germline cells once they leave the niche. However, overexpression of $d p p$ in somatic cells blocks germline differentiation $[11,13]$, suggesting the existence of a Dpp signaling threshold above which pathway activation can overcome endogenous antagonists.

Building upon our understanding of how the DppThickveins-phosphorylated Mad-Bam pathway controls GSC maintenance, the field is beginning to delve more deeply into how the ovarian niche first forms, how Dpp signaling from the niche is modulated and how the niche responds to environmental cues. Addressing these fundamental questions will provide a framework with which to better understand niches across species.

\section{Formation of the ovarian niche}

GSCs arise from primordial germ cells (PGCs) that first form at the posterior pole of the embryo. Through a series of migratory events, these PGCs make their way towards the gonadal mesoderm and eventually coalesce with a subpopulation of surrounding somatic cells to form the embryonic gonad [28]. Initially, about seven to 13 PGCs are incorporated into each gonad [29]. This number expands to approximately 100 by the end of larval development. Cell-cell communication involving the epidermal growth factor (EGF) pathway helps to coordinate the expansion of the germline with the surrounding gonadal mesoderm [30].

Transformation of the primitive gonad into an adult ovary begins during late larval development, starting with the formation of terminal filaments [31] (Figure 1). These structures are composed of eight to 10 disc-shaped cells that demarcate individual ovarioles in the developing ovary. They arise from small clusters of cells that organize themselves into stacks. The actin-depolymerizing factor Cofilin/ADF, encoded by the twinstar gene, regulates the actin cytoskeletal rearrangements that drive the intercalation of presumptive terminal filament cells 
[32]. Terminal filament formation occurs progressively, in a medial to lateral direction across the gonad [33]. The steroid hormone ecdysone or its metabolites probably govern the timing of these morphogenic events, as mutations in the ecdysone receptor or its binding partner ultraspiracle result in heterochronic defects and malformation of these structures [34].

While the mechanisms that designate specific somatic cell fates across the larval gonad remain unclear, enhancer trap screens revealed a small number of genes that exhibit high levels of expression in the developing terminal filament [33]. One of these genes, bric-a-brac $(b a b)$, encodes a $\mathrm{BTB} / \mathrm{POZ}$ domain transcription factor $[33,35]$. The expression of $b a b$ is first observed in the female gonad during late larval development and continues to mark terminal filament cells through adulthood. Disruption of $b a b$ results in terminal filament defects accompanied by severe morphological defects in the adult ovary, revealing that the overall organization of the adult ovary depends on proper terminal filament formation. A second transcription factor Engrailed also marks terminal filaments and is necessary for their development [36]. Identifying the transcriptional targets of Bab and Engrailed within the developing gonad remains important work for the future.

Cap cells, which help form the functional GSC niche in the adult ovary, are specified as the terminal filament formation nears completion. Cap cells can be distinguished based on a number of morphological and molecular markers. They form immediately adjacent to the posterior tips of the terminal filaments and express $b a b$, engrailed, hedgehog and high levels of Lamin C $[4,33,37,38]$, but are not incorporated into the growing terminal filament stack. Several studies have shown that the Notch pathway helps to promote cap cell formation $[39,40]$. Xie and colleagues showed that terminal filament cells express the Notch ligand Delta shortly after they begin to organize [39]. Delta activates Notch in adjacent somatic cells, inducing them to become cap cells. Overexpression of Delta or an activated form of Notch results in an accumulation of ectopic cap cells in the adult ovary. These extra cap cells are associated with ectopic GSCs, indicating that they act as functional niches. Heterozygous Notch mutant germaria carry a decreased number of cap cells, suggesting that Notch signaling is both necessary and sufficient for cap cell formation in the developing gonad. The expression of the E(spl)m7-LacZ Notch target reporter suggests that Notch signaling remains active in adult cap cells. Indeed, disruption of Notch specifically in adults leads to a decrease of cap cells within adult germaria over time and a subsequent reduction in the number of GSCs [39]. Overexpression of activated Notch in adult escort cells does not convert them into cap cells or result in ectopic niche formation, indicating that escort cell identity becomes set during pupal development. The basis for the stabilization of this cell fate remains uncharacterized.

\section{Stem cell capture by the niche}

Of the approximately 100 PGCs that populate each larval gonad, only a subset become GSCs while the rest differentiate to form germline cysts. The hallmarks of GSC selection become evident during the larval to pupal transition and involve a number of mechanisms. While germline cells of the larval gonad do not express bam, they differentiate in response to ectopic bam expression $[41,42]$. Moreover, all PGCs exhibit phosphorylated Mad expression prior to terminal filament formation, suggesting that BMP signaling blocks bam expression in larval gonads as it does in adults $[41,42]$.

Upon terminal filament formation, PGCs begin to exhibit spatially restricted changes in gene expression. In the posterior of the gonad, away from the terminal filaments, germline cells begin to express bam and show morphological signs of cyst development, while germline cells immediately adjacent to the terminal filament and newly established cap cells remain undifferentiated and express markers of Dpp signal responsiveness [42]. These cells, which probably give rise to adult GSCs, can undergo clonal expansion, giving rise to daughter GSCs that inhabit the same adult germarium. These findings suggest a simple model wherein PGCs immediately adjacent to cap cells receive BMP signals, continue to repress bam transcription and thus become incorporated into the maturing cap cell niche.

Additional enhancer trap and cell transplantation experiments suggest there may be a bias in which PGCs associate with the nascent niche and ultimately become GSCs [43]. This mechanism appears flexible, however, as the same PGC can give rise to cells located both inside and outside the niche during its initial formation. How Dpp production and responsiveness become restricted during the transition from the larval/pupal gonad to the adult ovary and how PGCs home in on the newly formed niches remain unclear.

\section{Modulation of adult niche signaling by the extracellular matrix}

Recent work has begun to characterize how the extracellular matrix modulates BMP signaling in the adult ovarian niche. For example, type IV collagen - encoded by the viking gene - regulates the distribution of Dpp and helps foster interactions between BMP ligands and their receptors in the embryo [44]. Disruption of viking results in a modest GSC expansion phenotype, suggesting that this extracellular matrix component restricts the spread of Dpp, thereby creating a very localized source of ligand at the anterior tip of the germarium (Figure 2). 
The division abnormally delayed (dally) gene, a member of the glypican family of integral membrane heparin sulphate proteoglycans [45], also plays a critical role in regulating the distribution and stability of Dpp within the ovarian GSC niche. Dally, like other heparin sulphate proteoglycans, is a component of the extracellular matrix and covalently attaches to the plasma membrane by glycosylphosphatidylinositol linkage [45]. Heparin sulphate proteoglycans act as co-receptors for a variety of secreted proteins such as Wnts, Fibroblast Growth Factors, Transforming Growth Factor beta and Hedgehog [46]. In Drosophila, Dally promotes the stability and transport of Dpp [47]. Dally is expressed in the cap cells, and dally mutants display a GSC loss phenotype accompanied by reduced Dpp signaling and premature expression of Bam within the germline [48,49]. In contrast, dally overexpression in somatic cells outside the niche results in an expansion of GSC-like cells $[14,48,49]$. While these findings show that the extracellular matrix modulates Dpp signaling within germaria, further work will be needed to elucidate the mechanisms that coordinate the deposition of extracellular matrix components within the niche and control their functions.

\section{Pathways that regulate niche signaling.}

Several additional molecules function in the niche, either through or in parallel to Dpp signaling. The genes female sterile (1) $Y b(Y b)$, hedgehog and piwi are expressed in somatic cells at the anterior tip of the germarium [37,50-53]. Loss of $Y b$, a large hydrophilic protein with limited homology to RNA helicases, disrupts the maintenance of both GSCs and follicle stem cells within the germarium [52,53]. Mutations in piwi, which encodes the founding member of a highly conserved family of proteins that function in various small RNA pathways, also cause a significant GSC loss phenotype. Overexpression of piwi within somatic cells of the germarium results in an expanded number of GSCs [50,51]. Hedgehog-mediated signaling primarily regulates follicle stem cells, but hedgehog mutants also exhibit a mild GSC loss phenotype $[37,38,53] . Y b$ mutants exhibit reduced hedgehog and piwi expression in terminal filament and cap cells [53]. Further genetic evidence suggests that $Y b$ regulates, through piwi-dependent and hedgehogdependent mechanisms, parallel pathways that control GSC and follicle stem cell maintenance, respectively. piwi appears to regulate GSCs in a $d p p$-independent manner [53], suggesting that additional unidentified GSC maintenance signals emanate from the cap cells.

Recent work shows that components of the Janus kinase/signal transducer and activator of transcription (Jak/Stat) pathway promote Dpp production by cap cells $[15,54,55]$. Overexpression of the Jak/Stat ligands unpaired and unpaired-2 in somatic cells results in GSC tumor formation, while mutations in pathway components cause a GSC loss phenotype [15,54,55]. Stat reporters show activation of the pathway in somatic cells at the anterior tip of the germarium, and clonal analysis reveals that pathway activation in cap cells is critical for GSC maintenance. Disruption of the Jak/Stat pathway does not affect terminal filament or cap cell formation and, unlike the Notch pathway, overactivation of the Jak/ Stat pathway during development does not result in ectopic cap cells. Transcript analysis shows that the Jak/ Stat pathway positively regulates $d p p$ mRNA levels, providing a simple model for how this pathway promotes GSC self-renewal $[15,55]$.

Several lines of evidence indicate that the germline itself can signal back to the surrounding somatic cells to regulate their signaling output. As described above, the EGF pathway functions to regulate PGC and somatic cell numbers in the developing gonad [30]. This pathway also functions in adult germaria. Disruption of the stem cell tumor gene results in the cell-autonomous failure of germline differentiation in both male and females [56]. Stem cell tumor protein shares sequence similarity with Rhomboid and proteins within this class act to cleave transmembrane EGF proteins in the Golgi, thereby creating a diffusible ligand. EGF ligands produced by germline cells in turn activate the EGF receptor-RASRAF-MEK-mitogen-activated protein kinase pathway in the surrounding somatic cells of the germarium. This activation of the EGF pathway limits the number of GSCs in germaria by repressing dally expression in escort cells [14]. In contrast, disruption of EGF signaling causes an increase of dally expression outside the normal niche, presumably resulting in a broader distribution of stable Dpp [14]. In effect, this feedback loop ensures that differentiating germline cysts experience lower levels of BMP signaling.

\section{Cell adhesion and cell competition in the adult niche}

Drosophila E-cadherin promotes stem cell maintenance by anchoring the GSCs to the cap cells [57]. Encoded by the shotgun (shg) gene, E-cadherin is highly enriched at the adherens junctions between the cap cells and GSCs. Armadillo, a $\beta$-catenin homolog, also localizes to these sites. The shotgun and armadillo mutant GSCs quickly leave the anterior of the germarium [57]. The findings that shotgun and armadillo mutant PGCs within the developing gonad exhibit reduced interactions with newly formed cap cells [57] and the observation that Ecadherin contributes to the age-dependent decline of adult GSCs [58] highlight the importance of cell adhesion in promoting interactions between stem cells and their niches throughout life.

Several studies have shown that individual GSCs compete for space within niches $[59,60]$. Whether a 
particular stem cell is more or less competitive often depends on expression levels of E-cadherin [59]. GSCs with relatively high levels of E-cadherin exhibit more competitiveness than neighboring cells and tend to have larger areas of contact with the cap cells. Bam, and its binding partner Benign gonial cell neoplasm [61], negatively regulate E-cadherin. The bam and benign gonial cell neoplasm mutant GSC clones express high levels of E-cadherin and outcompete the neighboring wild-type GSCs for the niche [59]. These results suggest that an important part of the GSC differentiation program may involve the rapid downregulation of genes involved in fostering cell-cell contacts between these stem cells and adjacent niche cells.

\section{Insulin signaling influences the niche}

Systemic factors that vary in response to diet and age play an important role in modulating niche output and stem cell responsiveness to niche signals. For example, insulin signaling contributes to the maintenance of the niche in adult ovaries. Activation of the insulin pathway through inhibition of FOXO by phosphatidylinositol 3-kinase activates Notch signaling in the cap cells [62]. Drosophila insulin receptor (dinr) mutants have a time-dependent cap cell loss phenotype, leading to a reduction of GSCs over time [63]. dinr mutants exhibit severely reduced Notch signaling, and expressing an activated form of Notch rescues the dinr mutant cap cell and GSC loss phenotypes. Moreover, insulin signaling influences Ecadherin levels at the junction between cap cells and GSCs as dinr mutant cap cells display decreased levels of E-cadherin, independent of Notch signaling. Steroid hormones also contribute to the formation and regulation of GSC maintenance $[64,65]$, suggesting that multiple systemic inputs impinge upon the niche during development and in adulthood.

\section{Programming inside and outside the niche}

Several studies have begun to reveal how epigenetic programming regulates the function and identity of somatic cells within the niche. For example, mutations in the gene encoding the chromatin-associated protein Corto suppress the GSC loss phenotype exhibited by piwi mutants [66]. Disruption of corto also restores hedgehog expression in $\mathrm{Yb}$ mutant germaria. Corto protein interacts with both Polycomb and trithorax group proteins, suggesting that these chromatin-associated proteins may influence $Y b$, piwi and hedgehog-mediated regulation of the niche.

Piwi and small piwi-interacting RNAs (piRNAs) play an essential role in programming chromatin within the germarium and in defending the germline against unwanted transposable element activity [67-70]. Recent results show that piRNA production is intimately linked with heterochromatin formation [70]. Functional analysis of eggless, a histone methyltransferase that acts to modify lysine 9 on histone $\mathrm{H} 3$ (H3K9), shows that this histone modification enzyme is needed for normal germline differentiation [70,71]. Loss of eggless results in sterility marked by the accumulation of undifferentiated germ cells, a reduction of piRNA production and a subsequent increase in transposable element levels. Interestingly, Eggless activity is required in both germ cells and in the surrounding escort cells. Vreteno, a tudor domaincontaining protein involved in piRNA production, is also required in both the germline and surrounding somatic cells [72]. Exploring the links between germline and somatic piRNA-mediated chromatin silencing and how they relate to the function of the niche will be important work for the future.

Loss of another chromatin-associated protein, the histone demethylase Lsd1, results in the formation of GSC tumors [73-75]. Lsd1 acts in a cell nonautonomous manner, and cell-specific knockdown experiments show that Lsd1 functions in escort cells to repress the expression of niche-specific signals [74]. Undifferentiated germ cells in Lsd1 mutants exhibit increased Dpp signaling, and reducing $d p p$ levels within escort cells suppresses the Lsd1 phenotype. The loss of Lsd1 during development results in the misexpression of cap cell markers in escort cells. While lineage tracing needs to be performed to determine whether cap cells and escort cells have a common precursor, the finding that escort cells can potentially express cap cell markers and vice versa suggests that these two cell populations may have similar developmental potential within the developing gonad $[13,39,42,74]$. Furthermore, these findings suggest that certain factors play a crucial role in limiting the size of the cap cell niche.

Lsd1 also functions to repress $d p p$ expression in adult escort cells independent of any changes in cell fate [74]. Whether Lsd1 directly targets the $d p p$ gene or some upstream regulator remains unknown. Lsd1 expression is ubiquitous within the germarium, and overexpression of an Lsd1 transgene in cap cells does not result in a stem cell loss phenotype (SE and MB, unpublished data). Lsd1 activity may therefore be spatially limited in some way or this histone demethylase could be recruited to specific sites by other proteins that have more cell-specific expression patterns. The characterization of Lsd1 function in escort cells reveals that the active repression of niche-specific signals outside the normal microenvironment may be essential for proper tissue homeostasis in certain contexts.

\section{Conclusions}

Over the past decade, the study of Drosophila GSCs has yielded a wealth of information about the fundamental principles that govern cellular niches, and the 
characterization of in vivo mammalian niches will certainly benefit from these lessons. Mammalian niches will probably share common features with Drosophila GSC niches, but perhaps they will also share common markers as well. Aside from these cross-species comparisons, many basic questions about niche biology remain. How does the signaling output of the niche change in response to environmental cues or to aging? How does metabolism affect the size of the niche? How do stromal cells inside and outside the niche interact with one another? How are niche cells specified and how is their fate stabilized? Do niche cells perform functions aside from producing localized signaling molecules? We can anticipate that the continued study of model stem cell systems will lead to a deeper understanding of the formation and function of niches across tissues and across species, improved tissue engineering, advances in regenerative medicine and insights into how perturbations in microenvironments contribute to human disease.

This article is part of a review series on Stem cell niche.

Other articles in the series can be found online at

http://stemcellres.com/series/stemcellniche

\section{Abbreviations}

Bab, Bric-a-brac; Bam, Bag of marbles; BMP, bone morphogenetic protein; Dad, Daughters against dpp; Dally, Division abnormally delayed; Dinr, Drosophila insulin receptor; Dpp, Decapentaplegic; EGF, epidermal growth factor; Gbb, Glass bottom boat; GSC, germline stem cell; JAK/STAT, Janus kinase/ signal transducer and activator of transcription; Mad, Mothers Against Dpp; PGC, primordial germ cell; piRNA, piwi-interacting RNA; RT-PCR, reverse transcriptase-polymerase chain reaction; YB, Female sterile (1) Yb.

\section{Competing interests}

The authors declare that they have no competing interests.

\section{Acknowledgements}

The authors would like to thank Jose Cabrera for his help with making the illustrations and Nevine Shalaby and Violaine Mottier for their critical comments on the manuscript. The present work is supported by grants from the March of Dimes (5FY0910), the Cancer Prevention Research Institute of Texas (RP100516), the National Institutes of Health (R01GM086647; T32GM008203) and the E.E. and Greer Garson Fogelson Endowment (UTSW Medical Center).

Published: 25 November 2011

\section{References}

1. Bonnet D, Dick JE: Human acute myeloid leukemia is organized as a hierarchy that originates from a primitive hematopoietic cell. Nat Med 1997, 3:730-737.

2. O'Brien CA, Kreso A, Jamieson CH: Cancer stem cells and self-renewal. Clin Cancer Res 2010, 16:3113-3120.

3. Schofield $R$ : The relationship between the spleen colony-forming cell and the haemopoietic stem cell. Blood Cells 1978, 4:7-25.

4. Xie T, Spradling AC: A niche maintaining germ line stem cells in the Drosophila ovary. Science (NY) 2000, 290:328-330.

5. Kiger AA, Jones DL, Schulz C, Rogers MB, Fuller MT: Stem cell self-renewal specified by JAK-STAT activation in response to a support cell cue. Science (NY) 2001, 294:2542-2545.

6. Tulina N, Matunis E: Control of stem cell self-renewal in Drosophila spermatogenesis by JAK-STAT signaling. Science (NY) 2001, 294:2546-2549.
7. de Cuevas M, Matunis EL: The stem cell niche: lessons from the Drosophila testis. Development 2011, 138:2861-2869.

8. Fuller MT, Spradling AC: Male and female Drosophila germline stem cells: two versions of immortality. Science (NY) 2007, 316:402-404.

9. Spradling AC: Developmental genetics of oogenesis. In Development of Drosophila melanogaster. Volume 1. Cold Spring Harbor, NY: Cold Spring Harbor Press; 1993:1-70.

10. Morris LX, Spradling AC: Long-term live imaging provides new insight into stem cell regulation and germline-soma coordination in the Drosophila ovary. Development 2011, 138:2207-2215.

11. Xie T, Spradling AC: Decapentaplegic is essential for the maintenance and division of germline stem cells in the Drosophila ovary. Cell 1998, 94:251-260.

12. Wharton KA, Thomsen GH, Gelbart WM: Drosophila 60A gene, another transforming growth factor beta family member, is closely related to human bone morphogenetic proteins. Proc Natl Acad Sci U S A 1991, 88:9214-9218

13. Song X, Wong MD, Kawase E, Xi R, Ding BC, MCCarthy JJ, Xie T: Bmp signals from niche cells directly repress transcription of a differentiationpromoting gene, bag of marbles, in germline stem cells in the Drosophila ovary. Development 2004, 131:1353-1364.

14. Liu M, Lim TM, Cai Y: The Drosophila female germline stem cell lineage acts to spatially restrict DPP function within the niche. Sci Signal 2010, 3:ra57.

15. Wang L, Li Z, Cai Y: The JAK/STAT pathway positively regulates DPP signaling in the Drosophila germline stem cell niche. J Cell Bio/ 2008, 180:721-728

16. lovino N, Pane A, Gaul U: miR-184 has multiple roles in Drosophila female germline development. Dev Cell 2009, 17:123-133.

17. Hudson JB, Podos SD, Keith K, Simpson SL, Ferguson EL: The Drosophila Medea gene is required downstream of $\mathrm{dpp}$ and encodes a functional homolog of human Smad4. Development 1998, 125:1407-1420.

18. Chen D, McKearin D: Dpp signaling silences bam transcription directly to establish asymmetric divisions of germline stem cells. Curr Bio/ 2003, 13:1786-1791.

19. Chen D, McKearin DM: A discrete transcriptional silencer in the bam gene determines asymmetric division of the Drosophila germline stem cell. Development 2003, 130:1159-1170.

20. McKearin D, Ohlstein B: A role for the Drosophila bag-of-marbles protein in the differentiation of cystoblasts from germline stem cells. Development 1995, 121:2937-2947.

21. McKearin DM, Spradling AC: bag-of-marbles: a Drosophila gene required to initiate both male and female gametogenesis. Genes Dev 1990, 4:2242-2251

22. Ohlstein B, McKearin D: Ectopic expression of the Drosophila Bam protein eliminates oogenic germline stem cells. Development 1997, 124:3651-3662.

23. Casanueva MO, Ferguson EL: Germline stem cell number in the Drosophila ovary is regulated by redundant mechanisms that control Dpp signaling. Development 2004, 131:1881-1890.

24. Kai T, Spradling A: An empty Drosophila stem cell niche reactivates the proliferation of ectopic cells. Proc Natl Acad Sci U S A 2003, 100:4633-4638

25. Podos SD, Hanson KK, Wang YC, Ferguson EL: The DSmurf ubiquitin-protein ligase restricts BMP signaling spatially and temporally during Drosophila embryogenesis. Dev Cell 2001, 1:567-578.

26. Xia L, Jia S, Huang S, Wang H, Zhu Y, Mu Y, Kan L, Zheng W, Wu D, Li X, Sun Q, Meng A, Chen D: The Fused/Smurf complex controls the fate of Drosophila germline stem cells by generating a gradient BMP response. Cell 2010, 143:978-990.

27. Harris RE, Pargett M, Sutcliffe C, Umulis D, Ashe HL: Brat promotes stem cell differentiation via control of a bistable switch that restricts BMP signaling Dev Cell 2011, 20:72-83.

28. Starz-Gaiano M, Lehmann R: Moving towards the next generation. Mech Dev 2001, 105:5-18.

29. Campos-Ortega JA, Hartenstein V: The Embryonic Development of Drosophila melanogaster. New York: Springer-Verlag; 1985.

30. Gilboa L, Lehmann R: Soma-germline interactions coordinate homeostasis and growth in the Drosophila gonad. Nature 2006, 443:97-100.

31. King RC, Aggarwal SK, Aggarwal U: The development of the female Drosophila reproductive system. J Morphol 1968, 124:143-166.

32. Chen J, Godt D, Gunsalus K, Kiss I, Goldberg M, Laski FA: Cofilin/ADF is required for cell motility during Drosophila ovary development and oogenesis. Nat Cell Biol 2001, 3:204-209. 
33. Sahut-Barnola I, Godt D, Laski FA, Couderc JL: Drosophila ovary morphogenesis: analysis of terminal filament formation and identification of a gene required for this process. Dev Biol 1995, 170:127-135.

34. Hodin J, Riddiford LM: The ecdysone receptor and ultraspiracle regulate the timing and progression of ovarian morphogenesis during Drosophila metamorphosis. Dev Genes Evol 1998, 208:304-317.

35. Godt D, Laski FA: Mechanisms of cell rearrangement and cell recruitment in Drosophila ovary morphogenesis and the requirement of bric a brac. Development 1995, 121:173-187.

36. Bolivar J, Pearson J, Lopez-Onieva L, Gonzalez-Reyes A: Genetic dissection of a stem cell niche: the case of the Drosophila ovary. Dev Dyn 2006 235:2969-2979

37. Forbes AJ, Lin H, Ingham PW, Spradling AC: hedgehog is required for the proliferation and specification of ovarian somatic cells prior to egg chamber formation in Drosophila. Development 1996, 122:1125-1135.

38. Forbes AJ, Spradling AC, Ingham PW, Lin H: The role of segment polarity genes during early oogenesis in Drosophila. Development 1996, 122:3283-3294.

39. Song X, Call GB, Kirilly D, Xie T: Notch signaling controls germline stem cell niche formation in the Drosophila ovary. Development 2007, 134:1071-1080.

40. Ward EJ, Zhou X, Riddiford LM, Berg CA, Ruohola-Baker H: Border of Notch activity establishes a boundary between the two dorsal appendage tube cell types. Dev Bio/ 2006, 297:461-470.

41. Gilboa $L$, Lehmann R: Repression of primordial germ cell differentiation parallels germ line stem cell maintenance. Curr Biol 2004, 14:981-986.

42. Zhu CH, Xie T: Clonal expansion of ovarian germline stem cells during niche formation in Drosophila. Development 2003, 130:2579-2588.

43. Asaoka M, Lin H: Germline stem cells in the Drosophila ovary descend from pole cells in the anterior region of the embryonic gonad. Development 2004, 131:5079-5089.

44. Wang $X$, Harris RE, Bayston LJ, Ashe HL: Type IV collagens regulate BMP signalling in Drosophila. Nature 2008, 455:72-77.

45. Nakato H, Futch TA, Selleck SB: The division abnormally delayed (dally) gene: a putative integral membrane proteoglycan required for cell division patterning during postembryonic development of the nervous system in Drosophila. Development 1995, 121:3687-3702.

46. Kirkpatrick CA, Selleck SB: Heparan sulfate proteoglycans at a glance. J Cell Sci 2007, 120:1829-1832

47. Akiyama T, Kamimura K, Firkus C, Takeo S, Shimmi O, Nakato H: Dally regulates Dpp morphogen gradient formation by stabilizing Dpp on the cell surface. Dev Bio/ 2008, 313:408-419.

48. Guo Z, Wang Z: The glypican Dally is required in the niche for the maintenance of germline stem cells and short-range BMP signaling in the Drosophila ovary. Development 2009, 136:3627-3635.

49. Hayashi Y, Kobayashi S, Nakato H: Drosophila glypicans regulate the germline stem cell niche. J Cell Bio/ 2009, 187:473-480.

50. Cox DN, Chao A, Baker J, Chang L, Qiao D, Lin H: A novel class of evolutionarily conserved genes defined by piwi are essential for stem cel self-renewal. Genes Dev 1998, 12:3715-3727.

51. Cox DN, Chao A, Lin H: piwi encodes a nucleoplasmic factor whose activity modulates the number and division rate of germline stem cells. Development 2000, 127:503-514.

52. King FJ, Lin $\mathrm{H}$ : Somatic signaling mediated by $\mathrm{fs}(1) \mathrm{Yb}$ is essential for germline stem cell maintenance during Drosophila oogenesis. Development 1999, 126:1833-1844.

53. King FJ, Szakmary A, Cox DN, Lin H: Yb modulates the divisions of both germline and somatic stem cells through piwi- and hh-mediated mechanisms in the Drosophila ovary. Mol Cell 2001, 7:497-508.

54. Decotto E, Spradling AC: The Drosophila ovarian and testis stem cell niches: similar somatic stem cells and signals. Dev Cell 2005, 9:501-510.

55. Lopez-Onieva L, Fernandez-Minan A, Gonzalez-Reyes A: Jak/Stat signalling in niche support cells regulates dpp transcription to control germline stem cell maintenance in the Drosophila ovary. Development 2008, 135:533-540.

56. Schulz C, Wood CG, Jones DL, Tazuke SI, Fuller MT: Signaling from germ cells mediated by the rhomboid homolog stet organizes encapsulation by somatic support cells. Development 2002, 129:4523-4534
57. Song X, Xie T: DE-cadherin-mediated cell adhesion is essential for maintaining somatic stem cells in the Drosophila ovary. Proc Natl Acad Sci USA 2002, 99:14813-14818.

58. Pan L, Chen S, Weng C, Call G, Zhu D, Tang H, Zhang N, Xie T: Stem cell aging is controlled both intrinsically and extrinsically in the Drosophila ovary. Cell Stem Cell 2007, 1:458-469.

59. Jin Z, Kirilly D, Weng C, Kawase E, Song X, Smith S, SchwartZ J, Xie T: Differentiation-defective stem cells outcompete normal stem cells for niche occupancy in the Drosophila ovary. Cell Stem Cell 2008, 2:39-49.

60. Rhiner C, Diaz B, Portela M, Poyatos JF, Fernandez-Ruiz I, Lopez-Gay JM, Gerlitz $\mathrm{O}$, Moreno E: Persistent competition among stem cells and their daughters in the Drosophila ovary germline niche. Development 2009, 136:995-1006.

61. Ohlstein B, Lavoie CA, Vef O, GateffE, McKearin DM: The Drosophila cystoblast differentiation factor, benign gonial cell neoplasm, is related to DExH-box proteins and interacts genetically with bag-of-marbles. Genetics 2000, 155:1809-1819.

62. Hsu HJ, Drummond-Barbosa D: Insulin signals control the competence of the Drosophila female germline stem cell niche to respond to Notch ligands. Dev Biol 2011, 350:290-300.

63. Hsu HJ, Drummond-Barbosa D: Insulin levels control female germline stem cell maintenance via the niche in Drosophila. Proc Natl Acad Sci U S A 2009, 106:1117-1121.

64. Ables ET, Drummond-Barbosa D: The steroid hormone ecdysone functions with intrinsic chromatin remodeling factors to control female germline stem cells in Drosophila. Cell Stem Cell 2010, 7:581-592.

65. Konig A, Yatsenko AS, Weiss M, Shcherbata HR: Ecdysteroids affect Drosophila ovarian stem cell niche formation and early germline differentiation. Embo J 2011, 30:1549-1562.

66. Smulders-Srinivasan TK, Szakmary A, Lin H: A Drosophila chromatin factor interacts with the Piwi-interacting RNA mechanism in niche cells to regulate germline stem cell self-renewal. Genetics 2010, 186:573-583.

67. Lin H, Yin H: A novel epigenetic mechanism in Drosophila somatic cells mediated by Piwi and piRNAs. Cold Spring Harb Symp Quant Biol 2008, 73:273-281.

68. Yin $\mathrm{H}$, Lin H: An epigenetic activation role of Piwi and a Piwi-associated piRNA in Drosophila melanogaster. Nature 2007, 450:304-308.

69. Brennecke J, Malone CD, Aravin AA, Sachidanandam R, Stark A, Hannon GJ: An epigenetic role for maternally inherited piRNAs in transposon silencing. Science (NY) 2008, 322:1387-1392.

70. Rangan P, Malone CD, Navarro C, Newbold SP, Hayes PS, Sachidanandam R, Hannon GJ, Lehmann R: piRNA production requires heterochromatin formation in Drosophila. Curr Biol 2011, 21:1373-1379.

71. Clough E, Moon W, Wang S, Smith K, Hazelrigg T: Histone methylation is required for oogenesis in Drosophila. Development 2007, 134:157-165.

72. Zamparini AL, Davis MY, Malone CD, Vieira E, Zavadil J, Sachidanandam R, Hannon GJ, Lehmann R: Vreteno, a gonad-specific protein, is essential for germline development and primary piRNA biogenesis in Drosophila. Development 2011, 138:4039-4050.

73. Di Stefano L, Ji JY, Moon NS, Herr A, Dyson N: Mutation of Drosophila Lsd1 disrupts $\mathrm{H} 3-\mathrm{K} 4$ methylation, resulting in tissue-specific defects during development. Curr Bio/ 2007, 17:808-812.

74. Eliazer S, Shalaby NA, Buszczak M: Loss of lysine-specific demethylase 1 nonautonomously causes stem cell tumors in the Drosophila ovary. Proc Natl Acad Sci U S A 2011, 108:7064-7069.

75. Rudolph T, Yonezawa M, Lein S, Heidrich K, Kubicek S, Schafer C, Phalke S, Walther M, Schmidt A, Jenuwein T, Reuter G: Heterochromatin formation in Drosophila is initiated through active removal of $\mathrm{H} 3 \mathrm{~K} 4$ methylation by the LSD1 homolog SU(VAR)3-3. Mol Cell 2007, 26:103-115.

76. de Cuevas M, Spradling AC: Morphogenesis of the Drosophila fusome and its implications for oocyte specification. Development 1998, 125:2781-2789.

doi:10.1186/scrt86

Cite this article as: Eliazer S, Buszczak M: Finding a niche: studies from the Drosophila ovary. Stem Cell Research \& Therapy 2011, 2:45. 